\title{
Care of Non COVID Patients in COVID-19 Era Piryani $S^{1}{ }^{1}$ Piryani RM, ${ }^{2}$ Dangal $\mathrm{G}^{3}$
}

\author{
${ }^{1}$ Public Health Consultant, \\ Karachi, Pakistan. \\ ${ }^{2}$ Department of Internal Medicine, \\ Universal College of Medical Sciences, \\ Bhairahawa, Nepal. \\ ${ }^{3}$ Department of Obstetrics and Gynecology, \\ Kathmandu Model Hospital, \\ kathmandu, Nepal.

\section{Corresponding Author} \\ Suneel Piryani \\ Public Health Consultant, \\ Karachi, Pakistan. \\ E-mail: suneel.piryani@gmail.com
}

\begin{abstract}
The consequences of the COVID-19 pandemic are extensive and far-reaching. Non COVID communicable diseases continue to spread and non-communicable diseases continue to progress. People may access healthcare facilities little bit late due to fear of contracting COVID-19 and present with severe symptoms, even with complications. Nepal has been facing dual burden of both non-communicable and communicable diseases. The number of COVID-19 patients has continuously been rising in Nepal since the start of May 2020. There is an anticipated surge of infectious disease such as malaria, dengue fever, enteric fever, scrub typhus, leptospirosis during summer and monsoon seasons in Nepal. There will be surge of cases of acute undifferentiated febrile illness (AUFI) during monsoon. As fever is one of the very common symptoms of COVID-19, so COVID-19 needs to be considered in differential diagnoses of acute undifferentiated febrile illness.
\end{abstract}

\section{KEY WORDS}

AUFI, COVID-19, Communicable disease, Nepal

\section{Citation}

Piryani S, Piryani RM, Dangal G. Care of Non COVID Patients in COVID-19 Era. Kathmandu Univ Med J. 2020; COVID-19 Special Issue 70(2):111-2.

\section{INTRODUCTION}

The knowledge regarding COVID-19 continue to unfold. As of last week of June 2020, only population based public health interventions including detect cases with reverse transcriptase polymerase chain reaction (RT-PCR) testing from COVID-19 suspects as per World Health Organization definition of suspect, isolate identified patients, trace contacts and quarantine them (if possible test them) continue social distancing and practice infection prevention and control measures. In hospital, moderatesevere and critical care patients are dealt which are around $15 \%$ and $5 \%$ of all COVID-19 patients respectively. ${ }^{1}$ Elderly and people with pre-existing non-communicable diseases (NCDs) such as cardiovascular disease, chronic respiratory disease, diabetes mellitus, cancer also appear to be more vulnerable to becoming severely ill with the virus. ${ }^{2}$
The effects of the COVID-19 pandemic are extensive and far-reaching, affecting not only the SARS-CoV-2 infected patients and healthcare professionals/workers involved in caring these patients, but also patients suffering from other diseases means Non COVID diseases. ${ }^{3}$ Safeguarding health care workers is essential from their safety point of view and also utilizing their services for Non-COVID related diseases. ${ }^{1}$ With overwhelming anticipated harm of COVID-19, it is obvious that other diseases will continue to progress during this pandemic. Increase in noncommunicable diseases in developing countries has put burden on already overloaded healthcare capacity of these countries in dealing communicable disease. 
Nepal is now facing a double-triple burden of diseases; non-communicable-existing as well as diseases emerged in last decade and emerging communicable diseases such as dengue fever, scrub typhus, influenza (H5N1 and H1N1), leptospirosis, enteric fever, Malaria. ${ }^{4}$

There is an expected surge of infectious disease during summer and monsoon seasons as usual in developing countries including Nepal. The number of COVID-19 cases has rapidly been increasing in Nepal since the beginning of May 2020. ${ }^{5}$

Figure 1. COVID-19 New Cases Reported in Nepal ${ }^{5}$

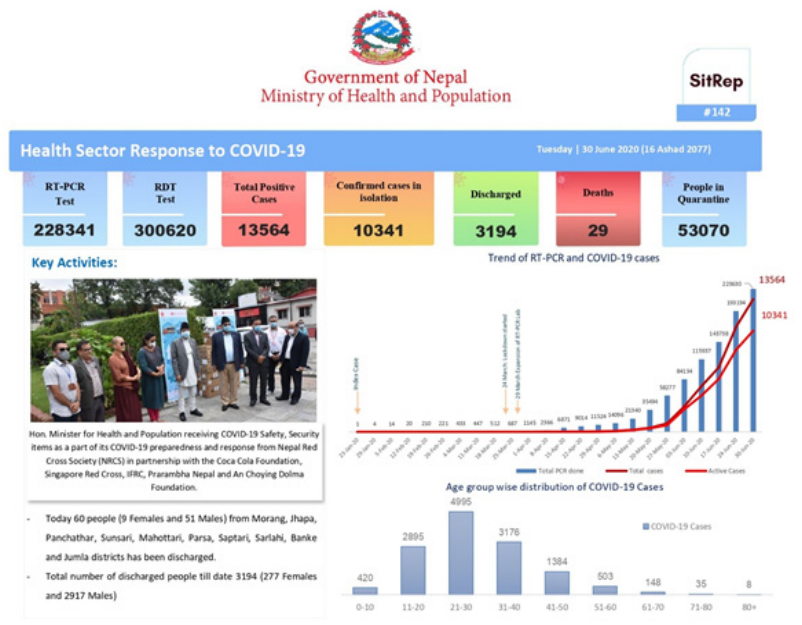

Figure 1. COVID-119 Situation update report \#142 as of 30 June 2020

\section{REFERENCES}

1. Hick JL, Biddinger PD. Novel Coronavirus and Old Lessons-Preparing the Health System for the Pandemic. N Engl J Med. May 14, 2020; 382 (20): e55. [DOI] https://doi.org/10.1186/s12879-019-4185-y

2. WHO I Information Note. COVID-19 and NCDs. World Health Organization. Available at: https://www.who.int/who-documentsdetail/covid-19-and-ncds. Accessed on May 21, 2020

3. Meszaros L. Navigating the healthcare system for non-COVID-19 patients MDLinx April 11, 2020. Avaialble at: https://www.mdlinx. com/article/navigating-the-healthcare-system-for-non-covid-19-pati ents/4tKq71KyfP5ZWwRGHxJ1or/ Accessed on May 21, 2020.

4. Rai SK. Changing Trend of Infectious Diseases in Nepal. Advances in Experimental Medicine and Biology. 2018; 1052:19-38. [DOI] DOI: 10.1007/978-981-10-7572-8_3.

5. Situation update report \#142 as of 30 June 2020 (Nepali and English Language) on health sector response to Coronavirus disease (COVID-19) Available at https://twitter.com/mohpnep/status/127795 6529178570754?lang=en Accessed on June 30, 202
We have to sort out what could likely be the best approach to treat these patients in frightening and uncertain environment of COIVD-19, else more people will die with other diseases than COVID-19 itself. These patients may also have fear of getting COVID-19 infection, so, we also have to listen their fear too and reduce their anxiety. May be fear of COVID-19 prevent them from presenting early at the health facility for treatment, so they may present late with severe disease or with complications, this will further increase the chances of mortality.

The significant number of patients presents with acute undifferentiated febrile illness (AUFI) during summer and monsoon period. In South Asia many pathogens cause AUFI such as malaria, dengue fever, enteric fever, scrub typhus, leptospirosis. AUFI leads to considerable morbidity and mortality among children, adults and elderly globally, more so in developing and under developed countries. The presentations and differential diagnoses of these diseases more or less are similar; formulating precise clinical diagnosis is challenging without laboratory confirmation. ${ }^{6,7}$

\section{CONCLUSION}

As fever is one of the very common symptoms of COVID-19. ${ }^{8,9}$ The number of cases has been increasing in Nepal since the beginning of May 2020 and may likely be exponential growth in days to come. The monson has already begun. There will likely be surge of infectious diseases too and also in cases of AUFI. We do believe COVID-19 must be considered in differential diagnose of AUFI.

6. Wangdi K, Kasturiaratchi K, Nery SV, Lau CL, Gray DJ, Clements ACA. Diversity of infectious aetiologies of acute undifferentiated febrile illnesses in south and Southeast Asia: a systematic review. BMC Infectious Diseases. 2019, 19:577 (DOI) https://doi.org/10.1186/ s12879-019-4185-y

7. Pokharel S, Karki M, Acharya B, Marasini B, Arjyal A. Outbreak of acute undifferentiated febrile illness in Kathmandu, Nepal: clinical and epidemiological investigation. BMC Infectious Diseases. (2020) 20:89 https://doi.org/10.1186/s12879-020-4803-8

8. Goyal P, Choi JJ, Pinheiro LC, et al. Clinical Characteristics of Covid-19 in New York City. N Engl J Med. 2020. [DOI] [PubMed] [DOI] doi: 10.1056/NEJMc2010419

9. Guan WJ, Ni ZY, Hu Y, et al. Clinical Characteristics of Coronavirus Disease 2019 in China. N Engl J Med. 2020; 382:1708. [PubMed] [DOI] DOI: 10.1056/NEJMoa2002032 